\title{
Education and informed decision making for prostate cancer screening in a high risk African American community
}

\author{
Puneet K Dhillon $^{1 *}$, Petros Grivas ${ }^{2,3}$, Brian Hobbs ${ }^{4}$, Daniel S Carlson ${ }^{5}$ and Samir Abraksia ${ }^{6}$ \\ ${ }^{1}$ Michigan State University College of Human Medicine, Department of Hematology/Oncology, Ascension Health-Providence Hospital and Medical Centers, USA \\ ${ }^{2}$ Department of Medicine, Division of Oncology, University of Washington (current), USA \\ ${ }^{3}$ Department of Hematology and Medical Oncology, Taussig Cancer Institute, Cleveland Clinic (during the study conduct), USA \\ ${ }^{4}$ Department of Quantitative Health Sciences, Lerner Research Institute, Cleveland Clinic, USA \\ ${ }^{5}$ Medicine Institute, Cleveland Clinic Foundation/South Pointe Hospital, USA \\ ${ }^{6}$ Department of Hematology and Medical Oncology, South Pointe Hospital, Taussig Cancer Institute, Cleveland Clinic, USA
}

\begin{abstract}
Background: Prostate cancer incidence and mortality in African Americans is higher vs Caucasians. Education programs and culturally appropriate outreach to highrisk groups in accordance with American Cancer Society (ACS) Informed Decision Making (IDM) guidelines are needed to reduce disparities. This study aims to examine whether IDM guidelines in a large high risk group setting can improve knowledge on prostate cancer and screening decisions.

Methods: Patients were included in a one-day outreach event and were given a pre and post- test focused on a standard informative educational PowerPoint and then were offered screening, prostate specific antigen and digital rectal exam (PSA + DRE). Pre and post- test number of correct answers were compared; patients were surveyed on their opinion on the program. The decision regarding screening after the intervention was tracked as well as the percent of prostate cancers diagnosed.

Results: 139 patients were included in the current analysis. Median number of correct answers at pre and post test was 9 and 12 with median improvement of +3 and $40 \%$ of patient showing improvement after education. $85 \%$ of patients indicated they wanted screening. Overall, $82 \%$ of patients favored IDM before screening. $73 \%$ of pts found the information "very helpful" (within a 5-point Likert scale) in decision-making.
\end{abstract}

Conclusions: Our education-based IDM led to significant improvement in knowledge about prostate cancer screening. Most patients preferred education prior to screening. Our approach paired with the use of navigation program is feasible and was positively received by a large high risk group.

\section{Introduction}

Prostate cancer is the most common cancer in American men, and the second leading cause of cancer-related death in men. The American Cancer Society (ACS) projected in 2018 there would be 164,690 of new cases and 29,430 deaths from prostate cancer [1]. The incidence of prostate cancer in African American men was found to be 1.6 times higher with the mortality rate being 2.4 times higher than in Caucasian men [2]. It was also observed that African American men are often diagnosed with more advanced stage and have poorer 5 -year survival [2].

African American (54.4\%) and Hispanic patients (42.3\%) are significantly less likely to report having had prostate specific antigen (PSA)-based screening than Caucasian patients (63.2\%). Healtheducation programs and culturally appropriate educational outreach efforts, especially targeted for high-risk groups, are needed to address such healthcare disparities [3].

Previous studies describe that most men did not know the key facts about prostate cancer screening (PCS). African Americans appeared less knowledgeable than Caucasians, but these differences were mediated by differences in educational level, health literacy, socioeconomic status and experience with PCS. Public health efforts to improve informed consent for PCS should focus on highlighting the relevant data and developing different approaches for men at different levels of education and experience with healthcare decisions [4].

Different national and international organizations have variable recommendations regarding PCS. The process of Informed Decision Making (IDM) is included in the guidelines of most organizations including the American Urology Association (AUA), European Association of Urology (EUA) and American College of Preventive Medicine. Most recently, the United States Preventative Services Task Force (USPSTF) has also aligned with individualized PCS decisions. Discussing the advantages and disadvantages of PCS has shown benefit in gaining knowledge and raising awareness in the African American men $[5,6]$. A church-based intervention to promote IDM for PCS

${ }^{\star}$ Correspondence to: Daniel S Carlson, Medicine Institute, Cleveland Clinic Foundation/South Pointe Hospital, Cleveland Clinic, 9500 Euclid Avenue, Cleveland, OH 44195, USA, Tel: (216) 956-0349; E-mail: carlsod2@ccf.org

Key words: prostate cancer screening, high risk population, African American, education, community outreach, informed decision making

Received: March 25, 2019; Accepted: April 08, 2019; Published: April 12, 2019 
among African-American men was reported to be a promising strategy [7].

ACS recommends that men who have at least a 10-year life expectancy should have an opportunity to make an informed decision with their physician regarding PCS after they receive information about the uncertainties, risks, and potential benefits associated with PCS [8]. Previous clinical investigation has found that physicians find it difficult to provide comprehensive, unbiased education about screening to patients [9]. Thus, encouraging men to participate in decision-making in the face of uncertainty about PCS represents a major challenge. This issue becomes especially thought-provoking when intervening among a group with an elevated risk of prostate cancer, and low health literacy $[5,7]$.

Using relevant decision aids, such as culturally sensitive written material, verbal information, and videos to educate patients about screening, can increase patient engagement and confidence in healthcare decisions $[8,10]$. However, the best way to educate the community through outreach programs is unclear. One study showed that more African American men participated in prostate cancer screening following patient navigator interventions than men who received a standard educational program [11]. Thus, the utility of patient navigation in this setting may be currently underutilized.

Another study addressed the challenges of IDM in prostate cancer community outreach to African American men and underlined the need for further research to assess the effectiveness of community-based outreach program [12]. Based on the current literature, it is evident that there is need for a PCS outreach program that can empower African American men with information and knowledge about screening choices and that invests in a patient advocate/navigator to ensure proper follow-up. Therefore, we hypothesized that a comprehensive educational intervention aiming to raise awareness among African American men about the rationale, risks and benefits of PCS would be perceived as beneficial and increase knowledge about PCS.

\section{Objectives}

This study aimed to create and evaluate a comprehensive, practical, effective educational model for prostate cancer education tailored for a high-risk population that can assist in PCS decision making process. Multiple measurable outcomes were captured and analyzed to assess model effectiveness. Furthermore, a patient navigation program was utilized to guide participants through education, screening decision and follow up care. Barriers and challenges in the implementation of this model and patient compliance were identified in order to inform future interventions.

\section{Methods}

The study was designed with a multi-step comprehensive approach covering the aspects of education including IDM components, screening options and process, patient navigation and close patient follow up.

\section{Participant selection}

This study was embedded within the context of a previously established community outreach program and specifically was implemented in multiple Men's Health events. Men for this project were recruited through The Cleveland Clinic Taussig Cancer Institute Outreach Program. Multiple channels were used, such as disseminating program information at local community centers, churches and church groups. The events were held at Cleveland Clinic facilities. Care was taken to disseminate the information in a consistent manner prior to each event across the entire local community areas to avoid selection bias. This study targeted the local community in the greater Cleveland, Ohio area, which included a predominantly African American population. However, since this study was conducted in the context of a larger health outreach program, other races and ethnicities were not excluded. The study was approved by the Cleveland Clinic IRB and supported by the Cleveland Clinic Taussig Cancer Institute Outreach Program and a grant from Genentech.

\section{Inclusion/Exclusion criteria}

Men over the age of 18 were eligible to participate in the educational component and men over the age of 40 were eligible for the screening component, which followed the educational component. Exclusion criteria included known history of prostate cancer or any other active malignancy, including treatment for any malignancy.

\section{Study design}

The study recruited patients as part of a yearly event which was a one-day program. The study was divided into three main components: Education for IDM, Screening, and Follow-up.

Education: All patients participated in the educational component of the study. Once the patients arrived to the day program, the education component composed of a pre-test, followed by an educational intervention, which included all aspects of IDM; a post-test was administered afterwards. IDM in our study was based on providing adequate education on prostate cancer, including benefits and risks. The pre- and post-tests included 15 questions, which tested participant knowledge of PCS, per ACS guidelines. Seven practicing experienced healthcare providers ranked 20 questions initially identified and the top 15 were selected as a standard measure to demonstrate knowledge about prostate cancer screening.

The education intervention was given in the form of a 20 -minute PowerPoint presentation that covered prostate cancer features and risks, method of screening [PSA, followed by digital rectal exam (DRE)], natural history of prostate cancer, risks and benefits of PCS. All components of IDM were embedded within this tool.

Screening: Following the pre-test, education/IDM, and post-test, patients were given the following options:

- Elect to be screened for prostate cancer with PSA and DRE

- Elect not to be screened for prostate cancer

- Unsure if they should be screened and prefer that a physician makes that decision

Screening was provided either on-site during the event or scheduled at a later time in an outpatient office if needed. Patients were then able to determine their comfort and satisfaction with their decision based on a 5-point Likert scale. They also were able to determine whether they would prefer to make their decision without receiving the education/ IDM intervention.

Patient navigation: Patients were followed closely after the event through an established navigation program. This program aimed to ensure that patients had appropriate follow up according to their screening results as well as regular follow up with primary care regardless of screening result.

Endpoints: Co-primary endpoints of the study were: (a.) the proportion of patients who demonstrated improved knowledge about PCS after the intervention, and (b.) to investigate the proportion of 
patients who found this educational intervention beneficial regarding PCS. Secondary endpoints included assessment of (a.) 10-year mortality of the participants, utilizing the University of California San Francisco (UCSF) mortality index, and its correlation with their decision about PCS, (b.) the proportion of men who chose to be screened, (c.) the proportion of men with abnormal screening results, (d.) the proportion of men with history of prostate cancer, (e.) the proportion of men who preferred screening without IDM, and (f.) the proportion of men who preferred that a physician should make the decision for them.

Data collection: This multistep interventional study was conducted in the context of pre-scheduled community outreach events, as noted above. Verbal understanding of the project and agreement with informed consent to participate was obtained from participants. Pre-printed data collection packets were distributed to participants with randomized identification numbers assigned to each participant. The packets included data collection tools that consisted of basic demographics, prostate cancer history and broader family history of cancer, assessment tool for 10-year mortality, educational pre-test, educational post-test and satisfaction survey as well as screening (PSA and DRE) results. Abnormal results from PSA and DRE screening were assessed based on National Comprehensive Cancer Network (NCCN) guidelines and patients were referred to a Urologist. If abnormal screening results warranted prostate biopsy, biopsy results were followed up and collected as well. All data were maintained in a properly secure database in a consistent manner.

The current NCCN guidelines for prostate cancer proposed that PSA testing should only be offered to men with a 10 or more-year life expectancy. Thus, we utilized the UCSF mortality index, which is a validated 12 question risk calculator to estimate 10-year survival.

\section{Analysis}

The objective of this study was to improve participant knowledge of PCS by evaluating the primary endpoint of the proportion of men who demonstrated improvement in PCS knowledge via preand post- tests. The other primary endpoint measured whether the intervention was helpful on a 5-point Likert scale where the two uppermost categories are considered a positive response. Improved understanding was defined as an increase in the number of correct answers seen on the post- vs. pre- test. It was anticipated that approximately 400 men would be enrolled and that at least 250 would have data available for analysis. Empirically the program was hypothesized to be successful with respect to an endpoint, if the lower boundary of the confidence interval was greater than $80 \%$. We pursued mixed effects logistic and linear regression to adjust for potential confounders, also considering the fact that data was derived from more than one events. A prespecified analysis was required for quality assurance after enrollment of at least the first 100 participants with plan to report the corresponding results. To better estimate the comparative benefit of IDM, however, the study was amended after enrolling 139 patients to include randomization to either IDM or shared decision making. Therefore, we hereby report the results of the study prior to the amendment and in list of the prespecified interim analysis.

\section{Results}

The presented data are restricted to the first two events in August $2015(\mathrm{n}=98)$ and April $2016(\mathrm{n}=41)$ with a total of 139 participants, as described above.
The majority of men in each session reported themselves as African American and therefore all analyses were performed twice once including all subjects and once restricted to African Americans. Results were similar for both analyses and although both analyses are summarized in the accompanying tables and figures only the "all subjects" analyses are discussed below. In addition, preliminary analyses showed no statistically significant differences with respect to subject characteristics or outcomes between the two events, and therefore those two events were combined for all analyses. Table 1 summarizes characteristics of men. Table 2 summarizes outcomes with primary endpoints and Table 3 summarizes outcomes with regard to secondary endpoints including screening decisions.

Overall, 93\% (127/137) of men self-reported as African American, and median age at the time of the event was 61 with $2 \%$ of subjects being less than 40 and $7 \%$ greater than 70 . Almost a third of men $(30 \%$, 28/92) with available data reported family history of prostate cancer. More than half of the subjects $(55 \%, 71 / 130)$ reported prior PSA assessment and most $(65 \%, 82 / 127)$ reported prior DRE. Half of men

Table 1. Subject characteristics

\begin{tabular}{|c|c|c|}
\hline & All Subjects & African-American Only \\
\hline Factor & $\mathrm{N}(\%)$ or Median (Range) & $\mathrm{N}(\%)$ or Median (Range) \\
\hline \multicolumn{3}{|l|}{ Race } \\
\hline AA & $127(93 \%)$ & - \\
\hline Caucasian & $8(6 \%)$ & - \\
\hline Other $^{1}$ & $2(1 \%)$ & - \\
\hline Age & $61(36-80)$ & $60(36-80)$ \\
\hline \multicolumn{3}{|l|}{ Education } \\
\hline Did not Graduate HS & $13(10 \%)$ & $13(10 \%)$ \\
\hline HS Graduate & $30(22 \%)$ & $29(23 \%)$ \\
\hline Some College & $56(41 \%)$ & $52(41 \%)$ \\
\hline College Graduate & $37(27 \%)$ & $32(25 \%)$ \\
\hline \multicolumn{3}{|l|}{ Employment } \\
\hline Employed & $82(62 \%)$ & $75(61 \%)$ \\
\hline Full Time & $61(77 \%)$ & $55(76 \%)$ \\
\hline Part Time & $18(23 \%)$ & $17(24 \%)$ \\
\hline Unemployed & $51(38 \%)$ & $48(39 \%)$ \\
\hline Looking & $8(19 \%)$ & $8(20 \%)$ \\
\hline Retired & $28(65 \%)$ & $25(62 \%)$ \\
\hline Disabled & $7(16 \%)$ & $7(18 \%)$ \\
\hline \multicolumn{3}{|l|}{ Family $\mathrm{Hx}$ of Prostate $\mathrm{Ca}$} \\
\hline No & $64(70 \%)$ & $58(67 \%)$ \\
\hline Yes & $28(30 \%)$ & $28(33 \%)$ \\
\hline \multicolumn{3}{|l|}{ Personal Hx of Prostate Ca } \\
\hline No & $119(93 \%)$ & $110(92 \%)$ \\
\hline Yes & $9(7 \%)$ & $9(8 \%)$ \\
\hline \multicolumn{3}{|l|}{$\begin{array}{l}\text { Doctor Has Previously } \\
\text { Discussed Screening }\end{array}$} \\
\hline No & $67(51 \%)$ & $62(51 \%)$ \\
\hline Yes & $64(49 \%)$ & $59(49 \%)$ \\
\hline \multicolumn{3}{|l|}{ Screening PSA in the Past } \\
\hline No & $59(45 \%)$ & $56(47 \%)$ \\
\hline Yes & $71(55 \%)$ & $64(53 \%)$ \\
\hline \multicolumn{3}{|l|}{ DRE in the Past } \\
\hline No & $45(35 \%)$ & $43(37 \%)$ \\
\hline Yes & $82(65 \%)$ & $74(63 \%)$ \\
\hline \multicolumn{3}{|l|}{ Primary Care Provider } \\
\hline No & $55(41 \%)$ & $52(42 \%)$ \\
\hline Yes & $78(59 \%)$ & $71(58 \%)$ \\
\hline
\end{tabular}

${ }^{1}$ Asian/Pacific Islander $-\mathrm{n}=1 ;$ “other" $-\mathrm{n}=1$ 
(63/125) reported that they had both; however, almost a third $(30 \%$, $38 / 125)$ reported they had neither. Most men $(59 \%, 78 / 133)$ indicated they had a primary care physician. For $28 \%(33 / 119)$ of men the only known risk factor for prostate cancer was their race.

Most men $(88 \%, 123 / 139)$ answered all 15 questions both prior to and after the education session. Eight-one percent (100/123) demonstrated an improvement in scores overall and this was consistent with predetermined endpoint of improvement greater than $80 \%$ determining program success. The median number of correct responses pre-test was 9 (range 2-15), with 34\% (45/134) of subjects answering fewer than half the questions correctly (counting "not sure" as an incorrect response). After the educational session however, the median number of correct responses increased to 12 (range $0-15$ ) with only $12 \%$ of subjects $(15 / 127)$ answering fewer than half the questions correctly. Over half $(57 \%, 72 / 127)$ of men answered correctly at least 12 questions and $10(8 \%)$ answered all 15 correctly in the post test. Overall, the median change in the number of correct responses was +3 (range -6 to

Table 2. Patient outcomes and Primary endpoints

\begin{tabular}{|c|c|c|}
\hline & All Subjects & African-American Only \\
\hline Factor & $\begin{array}{l}\text { N (\%) or Median } \\
\text { (Range) }\end{array}$ & $\mathrm{N}(\%)$ or Median (Range) \\
\hline \multicolumn{3}{|l|}{ Program Helpfulness } \\
\hline Not at all helpful & $-0-$ & $-0-$ \\
\hline Not helpful & $1(1 \%)$ & $1(1 \%)$ \\
\hline Neutral & $3(2 \%)$ & $3(2 \%)$ \\
\hline Helpful & $31(24 \%)$ & $27(22 \%)$ \\
\hline Very helpful & $96(73 \%)$ & $90(74 \%)$ \\
\hline \multicolumn{3}{|l|}{ No. Questions Answered (Pre-) } \\
\hline 5 & $1(1 \%)$ & $1(1 \%)$ \\
\hline 12 & $1(1 \%)$ & $1(1 \%)$ \\
\hline 14 & $3(2 \%)$ & $3(2 \%)$ \\
\hline 15 & $134(96 \%)$ & $122(96 \%)$ \\
\hline \multicolumn{3}{|l|}{ No. Questions Answered (Post-) } \\
\hline 0 & $2(1 \%)$ & $1(1 \%)$ \\
\hline 3 & $1(1 \%)$ & $-0-$ \\
\hline 13 & $1(1 \%)$ & $1(1 \%)$ \\
\hline 14 & $8(6 \%)$ & $8(6 \%)$ \\
\hline 15 & $127(91 \%)$ & $117(92 \%)$ \\
\hline \multicolumn{3}{|l|}{ No. Questions Answered (Pre-/Post) } \\
\hline May-15 & $1(1 \%)$ & $1(1 \%)$ \\
\hline $12 / 0$ & $1(1 \%)$ & $1(1 \%)$ \\
\hline $14 / 15$ & $3(2 \%)$ & $3(2 \%)$ \\
\hline $15 / 0$ & $1(1 \%)$ & $-0-$ \\
\hline 15-Mar & $1(1 \%)$ & $-0-$ \\
\hline $15 / 13$ & $1(1 \%)$ & $1(1 \%)$ \\
\hline $15 / 14$ & $8(6 \%)$ & $8(6 \%)$ \\
\hline $15 / 15$ & $123(88 \%)$ & $113(89 \%)$ \\
\hline No. Correct Responses (Pre-Test) ${ }^{2}$ & $9(2-15)$ & $9(2-15)$ \\
\hline$<8$ Correct Responses & $45(42 \%)$ & $39(32 \%)$ \\
\hline$\geq 12$ Correct Responses & $18(13 \%)$ & $15(12 \%)$ \\
\hline No. Correct Responses (Post-Test) ${ }^{2}$ & $12(0-15)$ & $12(0-15)$ \\
\hline$<8$ Correct Responses & $15(12 \%)$ & $15(13 \%)$ \\
\hline$\geq 12$ Correct Responses & $72(57 \%)$ & $67(57 \%)$ \\
\hline Pre-Test/Post-Test Improvement ${ }^{3}$ & $3(-6-9)$ & $3(-6-9)$ \\
\hline$\geq 4$ Point positive change & $54(40 \%)$ & $47(38 \%)$ \\
\hline No Improvement (0 point change) & $11(22 \%)$ & $11(23 \%)$ \\
\hline Worse score post-treatment & $19(14 \%)$ & $18(14 \%)$ \\
\hline
\end{tabular}

${ }^{1}$ Restricted to patients who completed the entire questionnaire

${ }^{2} \geq 14$ questions answered pre-test and $\geq 13$ questions answered post-test; positive changes indicate improvement in the number of correct responses; negative changes a decrease
Table 3 Patient outcome and secondary endpoints

\begin{tabular}{|c|c|c|}
\hline & All Subjects & African-American Only \\
\hline Factor & $\begin{array}{l}\mathrm{N}(\%) \text { or Median } \\
\text { (Range) }\end{array}$ & $\mathrm{N}(\%)$ or Median (Range) \\
\hline UCSF Mortality Index & $4(2-10)$ & $4(2-10)$ \\
\hline 2 & $33(28 \%)$ & $31(28 \%)$ \\
\hline 3 & $23(19 \%)$ & $21(19 \%)$ \\
\hline 4 & $17(14 \%)$ & $13(12 \%)$ \\
\hline 5 & $21(18 \%)$ & $20(18 \%)$ \\
\hline 6 & $10(8 \%)$ & $10(9 \%)$ \\
\hline 7 & $8(7 \%)$ & $7(6 \%)$ \\
\hline 8 & $4(3 \%)$ & $4(4 \%)$ \\
\hline 9 & $-0-$ & $-0-$ \\
\hline 10 & $3(3 \%)$ & $3(3 \%)$ \\
\hline \multicolumn{3}{|l|}{ Screening Decision } \\
\hline Do not screen & $13(10 \%)$ & $12(10 \%)$ \\
\hline Screen & $111(85 \%)$ & $104(87 \%)$ \\
\hline Let the provider & $6(5 \%)$ & $4(3 \%)$ \\
\hline \multicolumn{3}{|l|}{ Confidence in Screening Decision } \\
\hline Not at all confident & $1(1 \%)$ & $1(1 \%)$ \\
\hline Not confident & $-0-$ & $-0-$ \\
\hline Neutral & $5(4 \%)$ & $4(3 \%)$ \\
\hline Confident & $20(15 \%)$ & $15(12 \%)$ \\
\hline Very Confident & $105(80 \%)$ & $101(83 \%)$ \\
\hline \multicolumn{3}{|l|}{ Screening Occurred ${ }^{4}$} \\
\hline No & $24(17 \%)$ & $21(17 \%)$ \\
\hline Yes & $115(83 \%)$ & $106(83 \%)$ \\
\hline
\end{tabular}

${ }^{1}$ A subject was considered to have undergone screening if the subject had subsequent PSA and/or DRE

+9), while 40\% (54/135) of men demonstrated significant improvement (arbitrarily defined as an increase in the number of correct responses $>$ 4) while $8 \%(11 / 135)$ showed no change. The improvement in "scores" is shown graphically in Figure 1.

Overall, 97\% (127/131) of men categorized the program as helpful $(24 \%, 31 / 131)$ or very helpful $(73 \%, 96 / 131) ; 85 \%(111 / 130)$ indicated that they wanted screening $(83 \%, 115 / 139)$ actually went on to be screened, and 95\% (125/131) indicated that they felt confident $(15 \%$, $20 / 131)$ or very confident $(80 \%, 105 / 131)$ with their decision. Only $5 \%(6 / 130)$ preferred that a physician should make the screening decision for them despite the educational intervention. Interestingly, $15 \%(20 / 129)$ of participants preferred to have screening without the educational intervention.

The median UCSF mortality index score was 4 (range 2-10) among men with complete data $(\mathrm{n}=119)$; with $87 \%(104 / 119)$ having indices $<7$. The USCF mortality index demonstrated that scores of $>14$ leading to a $96 \%$ mortality at 10 years. For score of $0-6 ; 10$-year mortality ranges from $2.3-30 \%$. Thus, most participants demonstrated a low 10-year mortality score.

With respect to the other outcomes examined, there were no significant associations between the factors in Tables 2, 3 and whether men felt that the program was beneficial, and the confidence with which the decision to be screened was made. The decision to be screened was associated only with education, more likely to want screening if less educated $(p=0.04)$. Undergoing screening was associated with lower age $(\mathrm{p}=0.05)$, not having discussed screening with their doctor, $(\mathrm{p}=$ 0.01 ) and having neither PSA or DRE evaluation previously ( $\mathrm{p}=0.03$ ).

Overall, $88 \%$ (98/111 of those who chose screening) of participants underwent screening; 71\% (70/98) had both PSA and DRE (71/98 had 
A. All subjects

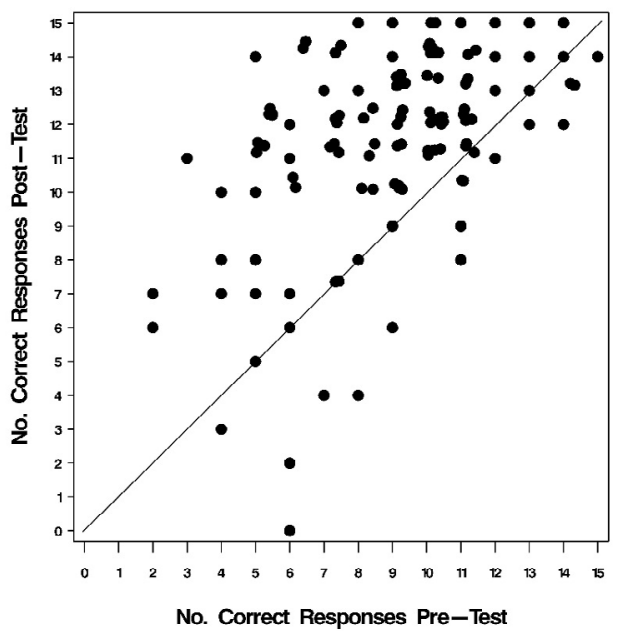

B. African-American subjects only

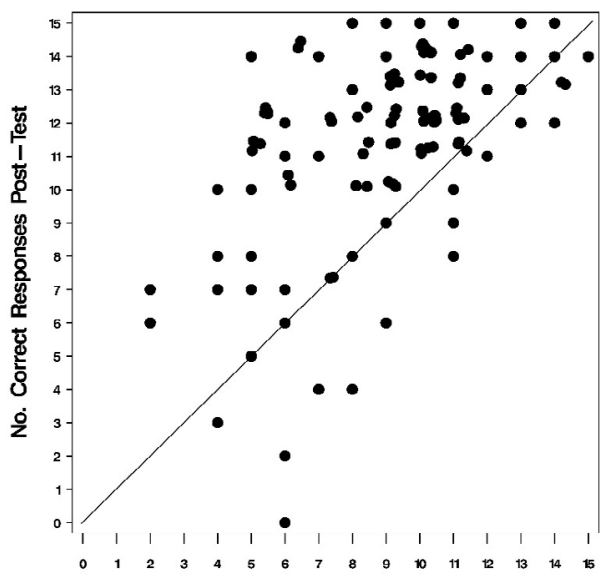

No. Correct Responses Pre-Test

Figure 1. Pre-test versus post-test scores ${ }^{1-}$

${ }^{1}$ Scatterplots comparing individual patient pre-test to post-test results. The reference line delineates patients observed to have improved performance in the post-test (above the line) from patients for whom the post-test resulted in fewer correct responses (below the line).

DRE, 97/98 had PSA testing) with 21\% (21/98) having either abnormal PSA or DRE which warranted follow up. Thirteen patients did not undergo screening due to various reasons, such as failure to follow up and the navigation program being unable to reach them. Eleven patients had follow-up with Urology and biopsies performed; out of those, 5 malignancies were identified.

\section{Discussion}

Raising prostate cancer awareness and continuing to empower patients to make an informed decision is a difficult task for most providers. This study aimed to create a program that can address prostate cancer disparities by empowering a high-risk population to make an informed decision on PCS. Most participants found this model to be beneficial and preferred information prior to making a decision about screening. Furthermore, most men chose to be screened and had improvement in their knowledge based on surveys before and after the educational intervention. The study has practical implications since there is a critical need for interventional studies across underserved populations. The study also demonstrated feasibility of such an infrastructure with positive feedback from the participants, almost all of whom found the educational program either helpful or very helpful.

A third of men reported a family history of prostate cancer. This is expected since we addressed a high-risk population with higher incidence of prostate cancer. The increasing knowledge about germline mutations that predispose to prostate and other cancers across populations is relevant and requires sophisticated educational strategies for raising awareness and education about genetic counseling, especially in high risk individuals even before diagnosis (e.g. based on personal and family history). Further investigation to explore the influence of this knowledge on the screening decision would be of high interest. This is also being discussed in the co-operative research group setting with considerations about larger consistent educational programs and relevant clinical trial designs.

There were numerous challenges and identified barriers for the implementation of our study program. Low health literacy, communication strategies, socio-economic and logistical factors, as well as previous negative experiences causing mistrust towards medical systems impacted patient compliance and decisions were identified. Moreover, these are very difficult to measure accurately. The high percentage of participants (41\%) who did not have a primary physician is not surprising since this event took place in an underserved community and underlines the need for a robust patient navigation program that ensures broader health education, proper follow up and recommended continuity of care. A small number of participants were difficult to contact or follow up due to incorrect phone numbers and address, change of address, or failure to show up to appointments after multiple attempts. However, the diligence of the navigation program attempted to minimize these barriers and suggested that the use of such a program is successful and vital.

Our study had several limitations, including the potential selection and confounding biases that can be patient-related and/or attributed to other potential concurrent interventions and events in the broader community. We tried to ensure consistency across the program logistics in the two events. Nearly all participants had longer than 10year expected mortality, which is appropriate for screening, however may suggest selection bias towards a motivated and relatively healthier patient population. Additionally, the higher screening rates observed than in the general African American population may also indicate bias from a motivate population. Our observed rate of abnormal PSA and DRE were significantly higher than published data in the general population [13]. However, whether this is due to bias or a true representation of high risk patients is not clear as there are little data for adequate comparison with our population. Lack of randomization to a control group significantly limits the interpretability of the results; however, we considered it unethical to include a group without educational intervention. Furthermore, we later amended the study to allow randomization between two interventional methods and the results will be reported in a subsequent publication. Only a small number of patients did not contribute data (missing answers) for the primary endpoints. The study was conducted in a single area mostly with African-American patients, thus the generalization of the results to other geographical areas and/or racial/ethnic populations may be limited. However, the study did provide a strong feasibility foundation for further programs and identified specific barriers that can inform such future initiatives.

\section{Conclusions}

Implementing a comprehensive program that utilizes patient navigators in an underserved, high risk community aiming to reinforce 
education and empower men to make their decision on PCS was very well received and feasible, resulting in increased topic-specific level of knowledge by the participants. Larger studies are being launched to validate our data and compare interventional strategies. These efforts align with the overarching need for broader, effective community outreach, optimal and equitable health care delivery and elimination of healthcare disparities. Engagement of multiple stakeholders remains critical.

\section{Acknowledgements}

The authors would like to thank the following individuals at the Cleveland Clinic Taussig Cancer Institute who have provided extensive research support:

Paola Raska, PhD; Kimberly Bell, Administrator, Taussing Cancer Institute; Devon Hickman, Outreach Program Manager; Sherry Wilson, PA-C; Georgia Shumpert, MSW, LSW; Kathy Smolenski, RN Research; Cathleen Hugney, RN, Manager, Research Program; Jacquelyn Bailey, PhD; Debra Allen, Patient Navigator; Amanda Hollis, Senior Director of Foundation Relations; Eileen Meisler, RN, Research; Jerry Sims, RN, MSN, Program Manager Community Outreach; Hui Zhu, MD; Jame Abraham, MD; Brian Bolwell, MD.

\section{Research support}

Foundation Grant for outreach program provided by Roche Genentech and institutional resources from the Cleveland Clinic.

\section{References}

1. American Cancer Society (2017) Cancer Facts \& Figures.

2. Merrill RM, Lyon JL (2000) Explaining the difference in prostate cancer mortality rates between white and black med in the United States. Urology 55: 730-735. [Crossref]
3. Hosain GM, Sanderson M, Du XL, Chan W, Strom SS (2011) Racial/Ethnic differences in predictors of PSA screening in a tri-ethnic population. Cent Eur J Public Health 19: 30-34. [Crossref]

4. Chan EC, Vernon SW, O'Donnell FT, Ahn C, Greisinger A, et al. (2003) Informed Consent for Cancer Screening with Prostate-Specific Antigen: How Well Are Men Getting the Message? Am J Public Health 93: 779-785. [Crossref]

5. Davis SN, Diefenbach MA, Valdimarsdottir H, Chen T, Hall SJ, et al. (2010) Pros and Cons of Prostate Cancer Screening: Associations with Screening Knowledge and Attitudes Among Urban African American Men. J Natl Med Assoc 102: 174-182. [Crossref]

6. Collins M (1997) Increasing Prostate Cancer Awareness in African American Men. Oncol Nurs Forum 24: 91-95. [Crossref]

7. Drake BF, Shelton RC, Gilligan T, Allen JD (2010) A church-based intervention to promote informed decision-making for prostate cancer screening among AfricanAmerican men. J Natl Med Assoc 102: 164-171 [Crossref]

8. Taylor KL, Williams RM, Davis K, Luta G, Penek S, et al. (2013) Decision making in prostate cancer screening using decision aids vs usual care: a randomized clinical trial. JAMA Intern Med 173: 1704-1712. [Crossref]

9. Wolf AM, Wender RC, Etzioni RB, Thompson IM, D'Amico AV, et al. (2010) American Cancer Society guideline for the early detection of prostate cancer: update 2010. $C A$ Cancer J Clin 60: 70-98. [Crossref]

10. Volk RJ, Hawley ST, Kneuper S, Holden EW, Stroud LA, et al. (2007) Trials of decision aids for prostate cancer screening: a systematic review. Am J Prev Med 33: 428-434. [Crossref]

11. O'Connor AM (1993) User Manual - Decisional Conflict Scale [document on the internet]. Ottawa: Ottawa Hospital Research Institute; copyright 1993 [updated 2010; cited 201603 02] p 16. Available from https://decisionaid.ohri.ca/docs/develop/User Manuals/UM_Decisional_Conflict.pdf

12. Volk RJ, Spann SJ, Cass AR, Hawley ST (2003) Patient Education for Informed Decision Making About Prostate Cancer Screening: A Randomized Controlled Trial with 1-Year Follow-Up. Ann Fam Med 1: 22-28. [Crossref]

13. Catalona WJ, Richie JP, Ahmann FR, Hudson MA, Scardino PT, et al. (1994) Comparison of digital rectal examination and serum prostate specific antigen in the early detection of prostate cancer: results of a multicenter clinical trial of 6,630 men. $J$ Urol 151: 1283-1290. [Crossref]

Copyright: (C2019 Dhillon PK. This is an open-access article distributed under the terms of the Creative Commons Attribution License, which permits unrestricted use, distribution, and reproduction in any medium, provided the original author and source are credited. 